\title{
Microminiature Fluidic Amplifier
}

\author{
Mark J. Zdeblick \\ Phillip W. Barth \\ Stanford University \\ Stanford, CA 94305
}

James B. Angell

\begin{abstract}
A microminiature fluidic amplifier with vertical-walled features $6 \mu \mathrm{m}$ wide and $35 \mu \mathrm{m}$ deep has been fabricated in silicon using dry anisotropic etching. Operation was demonstrated using nitrogen as the working fluid. DC gains greater than unity were seen over a supply pressure range of 10 to $80 \mathrm{psi}$. The amplifier is intended to be used as a circuit element in integrated fluidic circuits or, together with on-board electronics, as part of a transducer system. Dry etching techniques similar to those used here offer new opportunities for the micromachining of integrated sensors and actuators.
\end{abstract}

\section{Introduction}

A fluidic amplifier operates on gasses or liquids to amplify differential pressures or flows. A number of commercial fluidic systems, including sensors for high temperature, pressure, air speed, angular rate change, and fuel flow, are currently being used in various aerospace applications[1]. These commercial devices, made from non-semiconductor materials, are at least ten times larger in linear dimension than the device to be discussed. The smaller size of this new device results in higher operating pressures, and should lead to faster responses and a higher degree of integration than is currently available. Moreover, the demonstrated ability to integrate electronic function with silicon-based fluidic devices would make possible new sensors and actuators.

The device was made by anisotropically etching a $35 \mu \mathrm{m}$ deep trench in silicon and then sealing the trench with a brass manifold to form a capillary channel. This dry anisotropic etch is an extension of a recently developed dry etching technique developed by McVittie et al. [2] to etch depth $35 \mu \mathrm{m}$. The process yields near-vertical walls independent of crystal orientation, as shown in SEM photographs (Figures 1 and 2). The vertical etch rate of this mixed halocarbon $\left(\mathrm{SF}_{6}\right.$ and $\left.\mathrm{C}_{2} \mathrm{ClF}_{5}\right)$ etchant is $0.4 \mu \mathrm{m} / \mathrm{min}$ when used with the Drytek RIE-100, a parallel plate reactive ion etcher system. The etch depth of 35 $\mu \mathrm{m}$ is limited by the thickness of the photoresist.

The silicon wafer was also etched in $\mathrm{KOH}$ from the backside to provide feed-through holes for the input and output ports. Removable brass manifolds clamped on top and bottom of the silicon wafer provided ports accessible with Tygon tubing, while a $10 \mu \mathrm{m}$ layer of Parylene on the brass acted as gasket material.

A plan view of the wafer, shown in Figure 1, helps illustrate the operation of the device. The darker areas are the floor of the trench; the labels correspond to the different input and output ports. When the supply port is at a higher pressure than the vent, $P_{s}>P_{v}$, a laminar jet emanates from the supply's nozzle. This jet traverses the amplifier region and reaches the output ports, where it is split into two flows by the splitter. The input control ports steer the jet. When either a small differential input pressure, $\Delta P_{i}=P_{i_{r}}-P_{i_{l}}$, or input flow, $\Delta F_{i}$, is applied, the jet will be deflected toward one of the output ports, resulting in a differential output pressure $\&$ flow, $\Delta P_{o}$ $\& \Delta F_{o}$. For small variations about $\Delta P_{i}=0$, the device can be modeled as $\Delta P_{o}=A_{p} *\left(\Delta P_{i}+P_{i o}\right)$, where $A_{p}$ is the pressure gain of the device and $P_{i_{0}}$ is the input offset pressure. Ideally, $P_{i o}=0$.

The device was characterized by varying the supply pressure, $P_{s}$, and resistive loads, $R_{l}$, then measuring the input and output flow rates and pressures. From these data, we were able to calculate the following: pressure gain, $A_{p}$; flow gain, $A_{f}$; power gain, $A_{w}$.

\section{Results}

Of the four amplifiers fabricated, one has been thoroughly tested to date. This amplifier was tested by varying the capillary load resistance from 0 to $14 \mathrm{kPa} / \mathrm{sccm}$ and varying the supply pressures from 62 to $236 \mathrm{kPa}$ (9 to $34 \mathrm{psi}$ ). $A_{p}, A_{f}$, and $A_{w}$ were determined for each $P_{s}$ and $R_{l}$. $A_{f}$ decreases from 5.3 to 1.2 as $R_{l}$ increases from 0 to $14 \mathrm{kPa} / \mathrm{sccm}^{1}$. Operation at $P_{s}=133 \mathrm{kPa}(19 \mathrm{psi})$ produced the highest flow gain at any given $R_{l}$. The pressure gain increases asymptotically from 0.1 to 2.7 as $R_{l}$ increases. Again, operation at $P_{s}=133 \mathrm{kPa}(19$ psi) produced the highest gains. (Another amplifier produced a blocked output $\left(F_{o}=0\right)$ pressure gain of 4.5 , operating at 133 $\mathrm{kPa}$.) The power gain increases from zero to a maximum of 5.9 and then declines for higher $R_{l}$. The maximum power gain was observed at $R_{l} \approx 4 k P a / s c c m$ for most supply pressures. The $A_{f}$ vs. $A_{p}$ curve demonstrates some of the noise in the system. A set of smooth, parallel curves was expected; deviations are probably due to asymmetries in the load resistors.

The average flow rates in the supply, control, and output channels were also measured to determine the Reynolds number at both maximum flow and maximum power gain. The most striking observation is that the maximum output flow, $F_{o}$, is at most $31 \%$ of the supply flow, $F_{s}$, and that $F_{o}$ at maximum power gain is $21 \%$ of $F_{s}$. These figures imply that most of the supply flow is going out the vents, even when the output ports vent to air. A second observation is that the Reynolds number of the supply jet is between 400 and 1300 - near the turbulent flow transition. It should be noted that since $P_{s}$ is greater than twice the ambient pressure, supersonic flow at some point in the device is likely.

\section{Conclusions and projections}

This work demonstrated a $10 \mathrm{X}$ reduction in feature size and a $10 \mathrm{X}$ increase in $P_{s}$ compared to 'standard' fluidic amplifiers.

\footnotetext{
${ }^{3} \mathrm{sccm}$ is an abbreviation for standard cubic centimeters per minute
} 

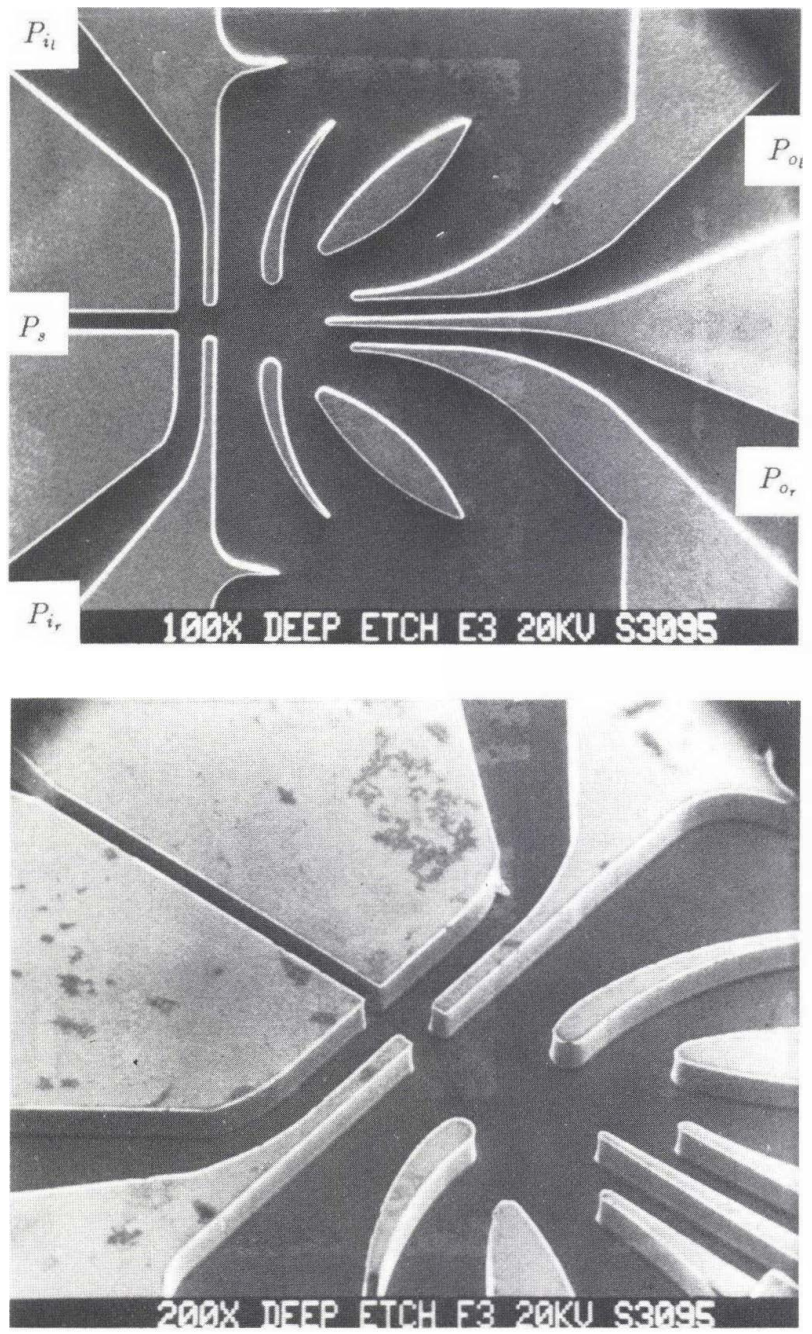

Power gain greater than one and input offset, $P_{i_{o}}$, less than $1 \%$ of the span of $P_{i}$ were demonstrated. In addition, this work shows that dry anisotropic etching can be reliably used to produce 'vertical-walled' structures with flat bottoms. Ring oscillator structures to obtain speed data have been fabricated and are awaiting testing.

It seems likely that improvements in single stage gains are obtainable with design optimization. Integration of pressure sensors and fluidic devices on a single wafer could provide fluidic to electronic transduction with low power and high speed. Compatible fabrication processes seem feasible, but need to be developed. Future devices could take advantage of developed anodic bonding techniques [3] to reduce leakage between channels and yield 'permanent' devices. It seems feasible to pursue a level of integration for fluidic devices comparable in size and complexity to 1960's vintage electronic circuits ${ }^{2}$.

\footnotetext{
${ }^{2}$ Mark J. Zdeblick is a Doctoral Candidate in the department of Electrical Engineering. His mailing address is Applied Electronics Laboratories Suite 16, Stanford University, Stanford CA, 94305.

Dr. Phillip W. Barth was with Stanford, he is now a Program Director at NovaSensor, 2975 Bowers Ave., Santa Clara, CA 95050.

Prof. James B. Angell is the Associate Chairman of the Department of Electrical Engineering at Stanford. His mailing address is McCullough 162, Stanford University, Stanford, CA, 94305.
}
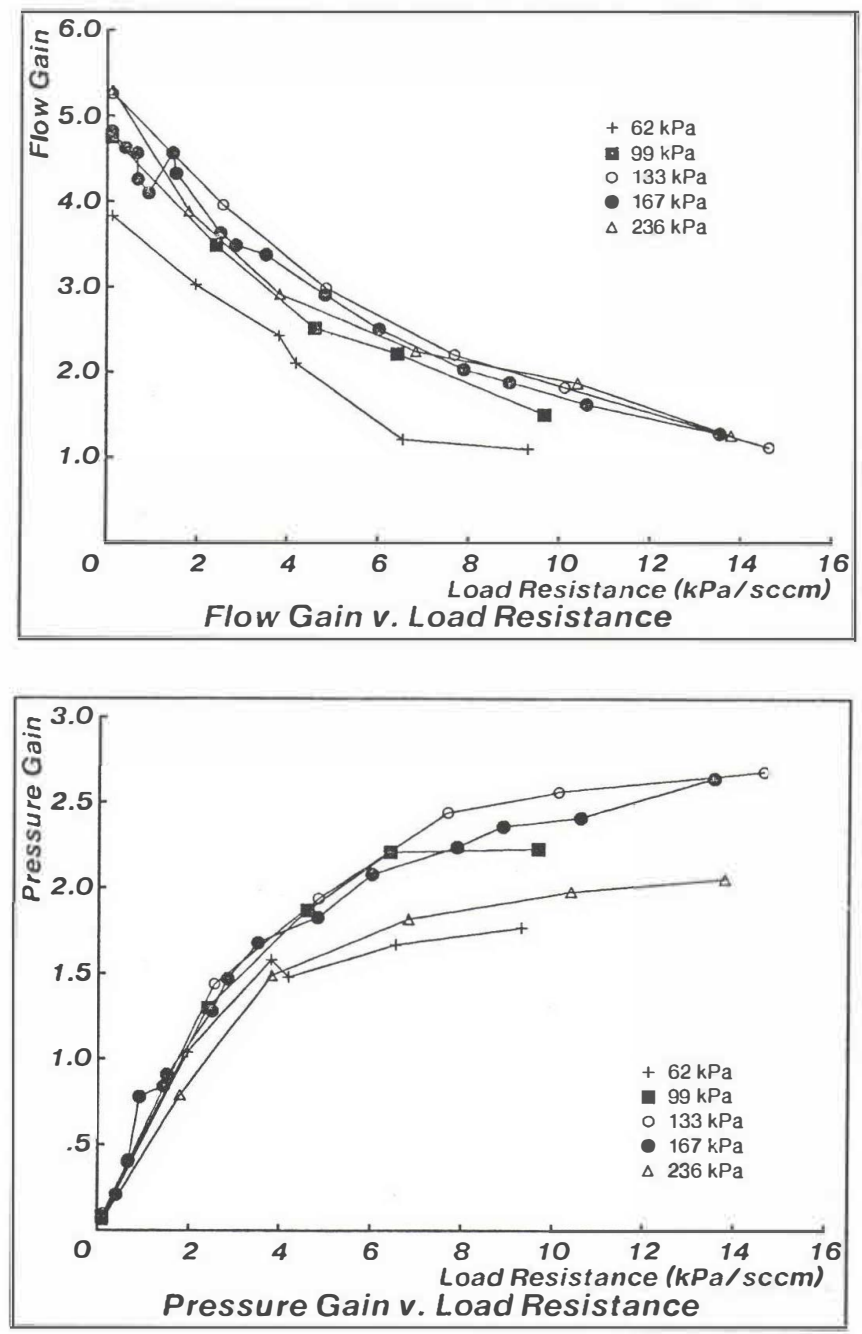

\section{Acknowledgements}

The authors would like to thank Dr. James McVittie of Stanford Integrated Circuits Laboratory for his help with the anisotropic etching technique, Martin Berdahl of Jet Propulsion Laboratories (JPL) for fabricating the brass test fixtures used to characterize the fluidic amplifier and for helpful discussions about fluidics in general, and Bill Reynolds for helpful discussions of fluid flow properties. This project was initially supported by JPL grant JPL 956888 and currently by General Motors grant GM 43-5.

\section{References}

[1] Garret Fluidic Systems Garret Pneumatic systems division, PO box 52-17, Phoenix, AZ 85010.

[2] "A nisotropic etching of $\mathrm{Si}$ using $\mathrm{SF}_{6}$ with $\mathrm{C}_{2} \mathrm{ClF}_{5}$ and other mixed halocarbons," J. McVittie and C. Gonzalez, Extended abstracts, Fall Meeting, Electrochemical Society, Vol 84-2, p 584.

[3] "A gas chromatography system fabricated on a silicon wafer using integrated circuit technology," Stephen Terry, Ph.D. Thesis, Stanford University Technical Report No. 4603-1, May 1975 